\title{
Diabetes Mellitus Accelerates Progression of Osteoarthritis in Streptozotocin-induced Diabetic Mice by Deteriorating Bone Microarchitecture, Bone Mineral Composition and Bone Strength of Subchondral Bone
}

\section{Hua-Jun Wang}

The First Clinical College, Jinan University \& Department of Orthopedic Surgery and Sports Medicine Center, The First Affiliated Hospital, Jinan University, Guangzhou, China

\section{Hugo Giambini}

Department of Biomedical Engineering, The University of Texas at San Antonio, San Antonio, TX, USA You-Jie Liu

The First Clinical College, Jinan University \& Department of Orthopedic Surgery and Sports Medicine Center, The First Affiliated Hospital, Jinan University, Guangzhou, China

\section{Ji-Wen Chen}

The First Clinical College, Jinan University \& Department of Orthopedic Surgery and Sports Medicine Center, The First Affiliated Hospital, Jinan University, Guangzhou, China

\section{Qiu-Shi Wang}

The First Clinical College, Jinan University \& Department of Orthopedic Surgery and Sports Medicine Center, The First Affiliated Hospital, Jinan University, Guangzhou, China

\section{Hui-ge Hou}

The First Clinical College, Jinan University \& Department of Orthopedic Surgery and Sports Medicine Center, The First Affiliated Hospital, Jinan University, Guangzhou, China

\section{Si-Min Luo}

The First Clinical College, Jinan University \& Department of Orthopedic Surgery and Sports Medicine Center, The First Affiliated Hospital, Jinan University, Guangzhou, China

\section{Jun-Yuan Chen}

The First Clinical College, Jinan University \& Department of Orthopedic Surgery and Sports Medicine Center, The First Affiliated Hospital, Jinan University, Guangzhou, China

\section{Teng-Feng Zhuang}

The First Clinical College, Jinan University \& Department of Orthopedic Surgery and Sports Medicine Center, The First Affiliated Hospital, Jinan University, Guangzhou, China

\section{Yuan-Feng Chen}


The First Clinical College, Jinan University \& Department of Orthopedic Surgery and Sports Medicine Center, The First Affiliated Hospital, Jinan University, Guangzhou, China

\section{Ting-Ting Wu}

The First Clinical College, Jinan University \& Department of Orthopedic Surgery and Sports Medicine Center, The First Affiliated Hospital, Jinan University, Guangzhou, China

\section{Zhen-gang Zha}

The First Clinical College, Jinan University \& Department of Orthopedic Surgery and Sports Medicine Center, The First Affiliated Hospital, Jinan University, Guangzhou, China

\section{Xiaofei Zheng ( $\sim 30442372 @ q q . c o m$ )}

Jinan University First Affiliated Hospital

\section{Research}

Keywords: Diabetes Mellitus, Osteoarthritis, Subchondral Bone, Bone Strength

Posted Date: March 27th, 2020

DOI: https://doi.org/10.21203/rs.2.24248/v2

License: (c) (i) This work is licensed under a Creative Commons Attribution 4.0 International License. Read Full License

Version of Record: A version of this preprint was published at Annals of Translational Medicine on May 1st, 2021. See the published version at https://doi.org/10.21037/atm-20-6797. 
The authors have withdrawn this preprint from Research Square 